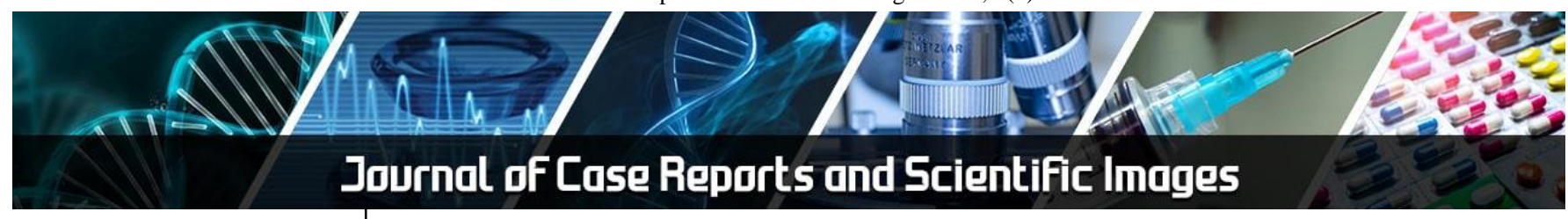

E-ISSN: 2708-0064

P-ISSN: 2708-0056

IJCRS 2020; 2(2): 12-16

http://www.allcasereports.com

Received: 15-04-2020

Accepted: 14-06-2020

\section{Dr. Vinod S}

Professor, Department of Radiology, Sree Mookambika Institute of Medical Sciences, Kulasekharam, Tamil Nadu, India

\section{Dr. Jagadeep MR}

Assistant Professor,

Department of Radiology, Sree

Mookambika Institute of

Medical Sciences,

Kulasekharam, Tamil Nadu, India

\section{Dr. Abdul Eksana AS}

Radiology Resident, Department of Radiology, Sree Mookambika Institute of Medical Sciences,

Kulasekharam, Tamil Nadu, India

\section{Corresponding Author:}

Dr. Vinod S

Professor, Department of

Radiology, Sree Mookambika Institute of Medical Sciences, Kulasekharam, Tamil Nadu, India

\section{Correlation between renal parenchymal thickness and EGFR in chronic kidney disease}

\author{
Dr. Vinod S, Dr. Jagadeep MR and Dr. Abdul Eksana AS
}

DOI: https://doi.org/10.22271/27080056.2020.v2.i2a.19

\begin{abstract}
Objective: A prospective study developed to determine the relationship between renal parenchymal thickness and glomerular filtration rate in patients with chronic kidney disease

Materials and Methods: From June 2019 to June 2020, 50 patients were clinically diagnosed with chronic kidney disease. The lowest serum creatinine was used for calculation of the glomerular filtration rate (eGFR) using the Modification of Diet in Renal Disease Study (MDRD) equations. The ultrasounds were done by a single radiologist and reviewed by 2 consultants. The renal parenchymal thickness was measured in the longitudinal from the renal sinus to the outer renal cortical margin at upper, mid and lower levels of each kidney and the mean value was estimated for each kidney. Statistical Package for Social Science (SPSS 20.0) version used for data analysis and an unpaired test was applied to find the statistical significance. Pearson correlation test applied to find the correlation between the variables. A P value less than $0.05(\mathrm{p}<0.05)$ was considered statistically significant at $95 \%$ confidence interval.

Results: US findings revealed that the mean renal parenchymal thickness was $11.2 \pm 3.01 \mathrm{~mm}$. $68 \%$ (Right) and $70 \%$ (Left) of cases demonstrated grade II echogenicity followed by grade III parenchymal echogenicity with poor cortico-medullary differentiation of $14 \%$ cases on right side and $12 \%$ on left side. Mean serum creatinine was 3.9 \pm 2.2 . The mean eGFR was $22.8 \pm 15.1$ using MDRD formula. There was a statistically significant relationship between renal parenchymal thickness and eGFR using MDRD formula ( $\mathrm{p}=0.002)$.

Conclusion: Renal parenchymal thickness measured on ultrasound appears closely related to the eGFR using MDRD formula. Therefore, renal parenchymal thickness should be considered in routine ultrasound reporting for the patients with chronic kidney disease who are not on dialysis.
\end{abstract}

Keywords: Chronic kidney disease, parenchymal thickness, estimated glomerular filtration rate, modification of diet in renal disease study

\section{Introduction}

The Chronic Kidney Disease (CKD) is defined as the abnormality of renal function or renal structure which present for more than 3 months duration. The CKD is considered to be the 6th deadliest disease in the world causing 2.4 million deaths per year. In the year 2019, around 850 million people are found to have chronic kidney disease worldwide and in India the patients with CKD remains undocumented and unregistered.

Currently, two main methods exist to evaluate and monitor kidney disease; these are conventional serum tests (eg; Serum creatinine) or biopsy. Among imaging techniques, magnetic resonance imaging is used to assess cortico-medullary differentiation ${ }^{[1]}$; however it is not a viable option in patients with an incompatible pacemakers or those who are claustrophobic. The use of gadolinium-based contrast in MRI may also lead to contrast induced nephrogenic systemic fibrosis ${ }^{[2]}$. The same can be said about contrast-enhanced computed tomography which adds the risk of significant radiation exposure, particularly in the young ${ }^{[3]}$.

Ultrasonography is one of the ideal methods for imaging kidneys and has been considered as the first imaging of choice in assessing CKD. The renal cortex, medulla and collecting system have different acoustic properties, so the pathological changes are easily discernible and can correlated to histological findings. Kidney measurements like pole-to- pole length, width and parenchymal thickness are associated with renal function and help in the better assessment of Chronic Kidney Disease status ${ }^{[4]}$. Renal cortical echogenicity plays an important role in diagnosis of interstitial fibrosis due to increased collagen deposit as this increases the cortical echogenicity ${ }^{[5]}$. Serial evaluation of renal length, cortical thickness and parenchymal echogenicity is helpful in identifying the progression of renal disease ${ }^{[6]}$. 
The renal length and parenchymal thickness decreases and parenchymal echogenicity increases in the process of CKD [7]. Renal length less than $10 \mathrm{~cm}$ associated with an increased echogenicity points to irreversible damage ${ }^{[8]}$. Renal artery spectral Doppler imaging is also performed to assess the renal hemodynamics associated with the alterations in the renal parenchyma and kidney function ${ }^{[9]}$. The Glomerular filtration rate is estimated by the Chronic Kidney Disease MDRD formula (Modification of Diet in Renal Disease study) ${ }^{[10]}$. MDRD formula estimates the GFR adjusted for body surface area and it is considered to be more accurate than the 24 hour urine collection creatinine clearance test by Cockcroft-Gault formula.

\section{Materials and Methods}

A cross sectional study, prospective study was conducted on patients with established chronic kidney disease referred to the department of Radiodiagnosis in our institution. The study was approved by the ethics committee. CKD patients of renal replacement therapy, patients with autosomal dominant polycystic kidney disease, renovascular nephropathy, acute kidney injury, solitary kidney, and pelvic cysts or renal tumour were excluded from the study. A total of 50 patients with CKD undergoing clinical followup with serum creatinine collected over the previous one year were referred for renal ultrasound. Anthropometric data (height and weight, measured as meters and kilograms, respectively) were recorded, and a peripheral blood sample was collected to measure serum creatinine using a modified kinetic Jaffe reaction. The eGFR was calculated by using MDRD equation as follows: $186 \mathrm{x}$ (creatinine / 88.4) -1.154 $\mathrm{x}$ (age) $-0.203 \mathrm{x}(0.742$ if female) $\mathrm{x}$ (1.210 if black).

All examinations were performed in the outpatient setting using standard gray scale B-mode imaging with a 5.0MHZ wide frequency band, phased assay probe (Siemens ACUSON X300). The parenchymal thickness was measured in the longitudinal plane from the renal sinus to the outer renal cortical margin at upper, mid and lower levels of each kidney perpendicular to the capsule. The mean parenchymal thickness was calculated for each kidney.

\section{Statistical analysis}

The data was expressed in number, percentage, mean and standard deviation. Statistical Package for Social Science (SPSS 20.0) version used for analysis. Unpaired test applied to find the statistically significant. Pearson correlation test applied to find the correlation between the variables. $\mathrm{P}$ value less than $0.05(\mathrm{p}<0.05)$ considered statistically significant at $95 \%$ confidence interval.

Table 1: Age distribution of cases with Chronic Kidney Disease

\begin{tabular}{|c|c|c|}
\hline Age (Years) & Number & Percentage (\%) \\
\hline$\leq 20$ yrs & 1 & 2.00 \\
\hline $21-40$ yrs & 5 & 10.00 \\
\hline $41-60$ yrs & 32 & 64.00 \\
\hline $61-80$ yrs & 10 & 20.00 \\
\hline$>80$ yrs & 2 & 4.00 \\
\hline
\end{tabular}

Table 2: Gender distribution of cases with Chronic Kidney Disease

\begin{tabular}{|c|c|c|}
\hline Gender & Number & Percentage (\%) \\
\hline Male & 31 & 62.00 \\
\hline Female & 19 & 38.00 \\
\hline
\end{tabular}

Table 3: Clinical etiology of Chronic Kidney Disease

\begin{tabular}{|c|c|c|}
\hline Causes of CKD & Number & Percentage (\%) \\
\hline Unknown etiology & 10 & 20.00 \\
\hline Diabetes mellitus alone & 6 & 12.00 \\
\hline Hypertension alone & 8 & 16.00 \\
\hline DM \& hypertension combined & 22 & 44.00 \\
\hline Other known causes & 4 & 8.00 \\
\hline
\end{tabular}

Table 4: Demographic characteristics and laboratory measurements of patients according to CKD stages

\begin{tabular}{|c|c|c|c|c|c|}
\hline Observation & Total $(\mathbf{n = 5 0})$ & CKD5 $(\mathbf{n = 1 9})$ & CKD4 (n=16) & CKD3 (n=13) & CKD2 (n=2) \\
\hline Age (years) & $53.9 \pm 14.8$ & $59.4 \pm 13.3$ & $53.1 \pm 13.1$ & $50.4 \pm 14.9$ & $30.5 \pm 21.9$ \\
\hline Gender(F/M) & $19 / 31$ & $8 / 11$ & $8 / 8$ & $2 / 11$ & $1 / 1$ \\
\hline Height (cm) & $159.2 \pm 12.2$ & $157.2 \pm 11.6$ & $159.1 \pm 11.8$ & $165.4 \pm 10.6$ & $138.5 \pm 2.1$ \\
\hline Weight (kg) & $58.8 \pm 14.3$ & $54.3 \pm 11.7$ & $59.3 \pm 15.6$ & $67.6 \pm 12.1$ & $40.5 \pm 7.7$ \\
\hline BMI (kg/m2) & $23.0 \pm 3.3$ & $21.0 \pm 2.8$ & $24.4 \pm 3.0$ & $23.2 \pm 3.7$ & $22.0 \pm 3.1$ \\
\hline Diabetes mellitus & 28 & 11 & 9 & 8 & - \\
\hline Hypertension & 30 & 13 & 9 & 8 & - \\
\hline Other known causes & 4 & - & 3 & 1 & - \\
\hline Unknown etiology & 10 & 4 & 3 & 1 & 2 \\
\hline Creatinine & $3.9 \pm 2.2$ & $6.4 \pm 1.6$ & $2.9 \pm 0.5$ & $1.95 \pm 0.2$ & $1.3 \pm 0.4$ \\
\hline e-GFR & $22.8 \pm 15.1$ & $9.0 \pm 2.2$ & $21.3 \pm 3.7$ & $38.6 \pm 8.4$ & $62.5 \pm 0.7$ \\
\hline Parenchymal thickness (mm) & $11.2 \pm 3.01$ & $10.2 \pm 3.2$ & $10.4 \pm 1.8$ & $13.2 \pm 2.6$ & $14.1 \pm 2.1$ \\
\hline
\end{tabular}

Table 5: Correlation between eGFR and renal parenchymal thickness

\begin{tabular}{|c|c|c|c|}
\hline Observation & Parenchymal thickness (right) & Parenchymal thickness (left) & Mean renal parenchymal thickness \\
\hline e-GFR & 2.2 & 2.3 & 0.54 \\
\hline p value & 0.0001 & 0.004 & 0.002 \\
\hline
\end{tabular}

Table 6: Analysis of receiver operating characteristics curve (eGFR $<60 \mathrm{~mL} / \mathrm{min}$ )

\begin{tabular}{|c|c|c|c|c|c|c|c|c|}
\hline Parameters & $\begin{array}{c}\text { Cut off } \\
\text { value }\end{array}$ & $\begin{array}{c}\text { AUC (95\% } \\
\text { confidence interval) }\end{array}$ & $\begin{array}{c}\text { Sensitivity } \\
(\%)\end{array}$ & $\begin{array}{c}\text { Specificity } \\
(\%)\end{array}$ & $\begin{array}{c}\text { Positive predictive } \\
\text { value }(\%)\end{array}$ & $\begin{array}{c}\text { Negative predictive } \\
\text { value }(\%)\end{array}$ & $\begin{array}{c}\text { Test } \\
\text { validity }\end{array}$ & p value \\
\hline $\begin{array}{l}\text { Parenchymal } \\
\text { thickness }\end{array}$ & 12.78 & $0.67(0.62-0.76)$ & 54 & 81 & 91.21 & 61.28 & 71.24 & 0.001 \\
\hline
\end{tabular}




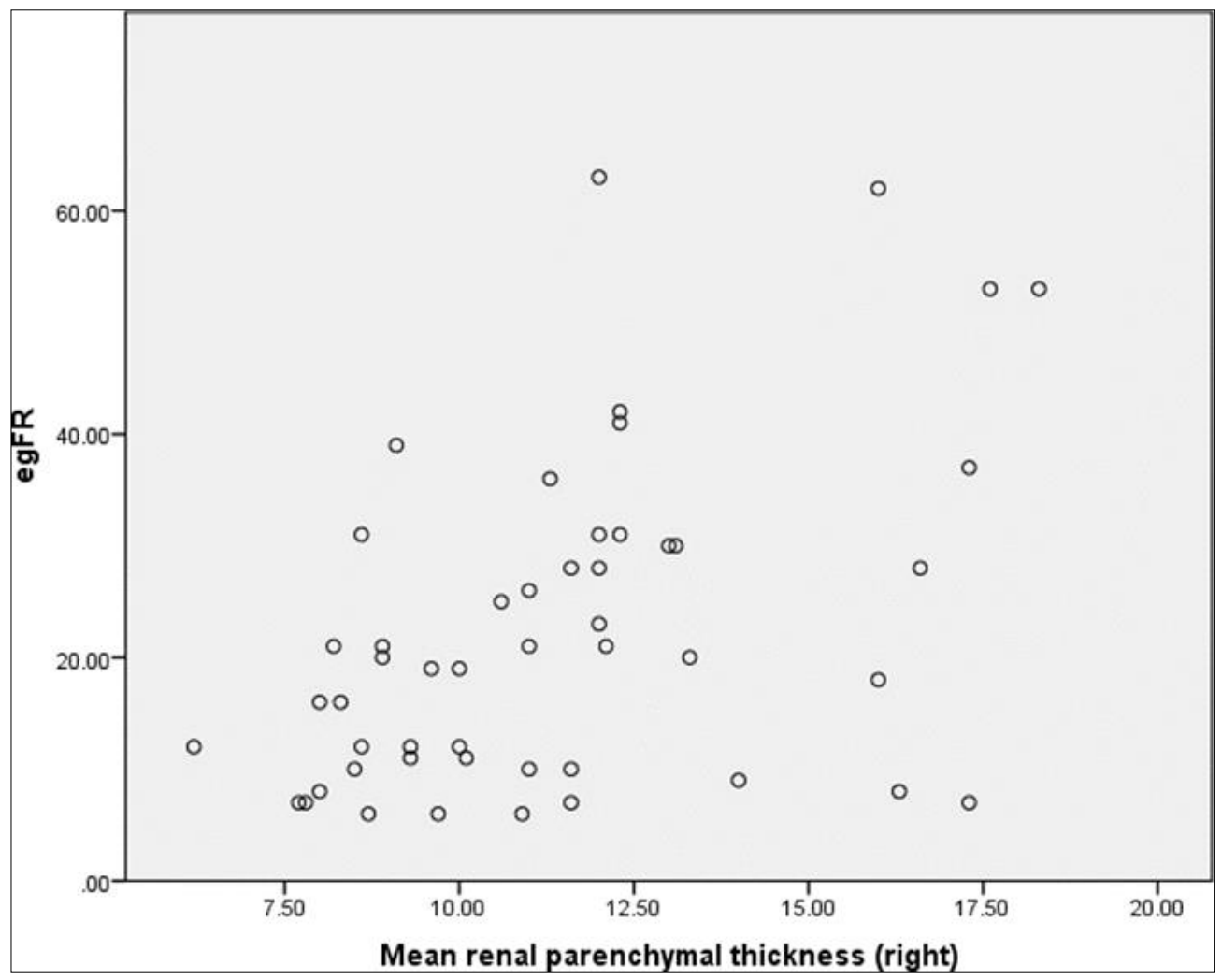

Fig 1: Correlation between eGFR and mean renal parenchymal thickness (right kidney)

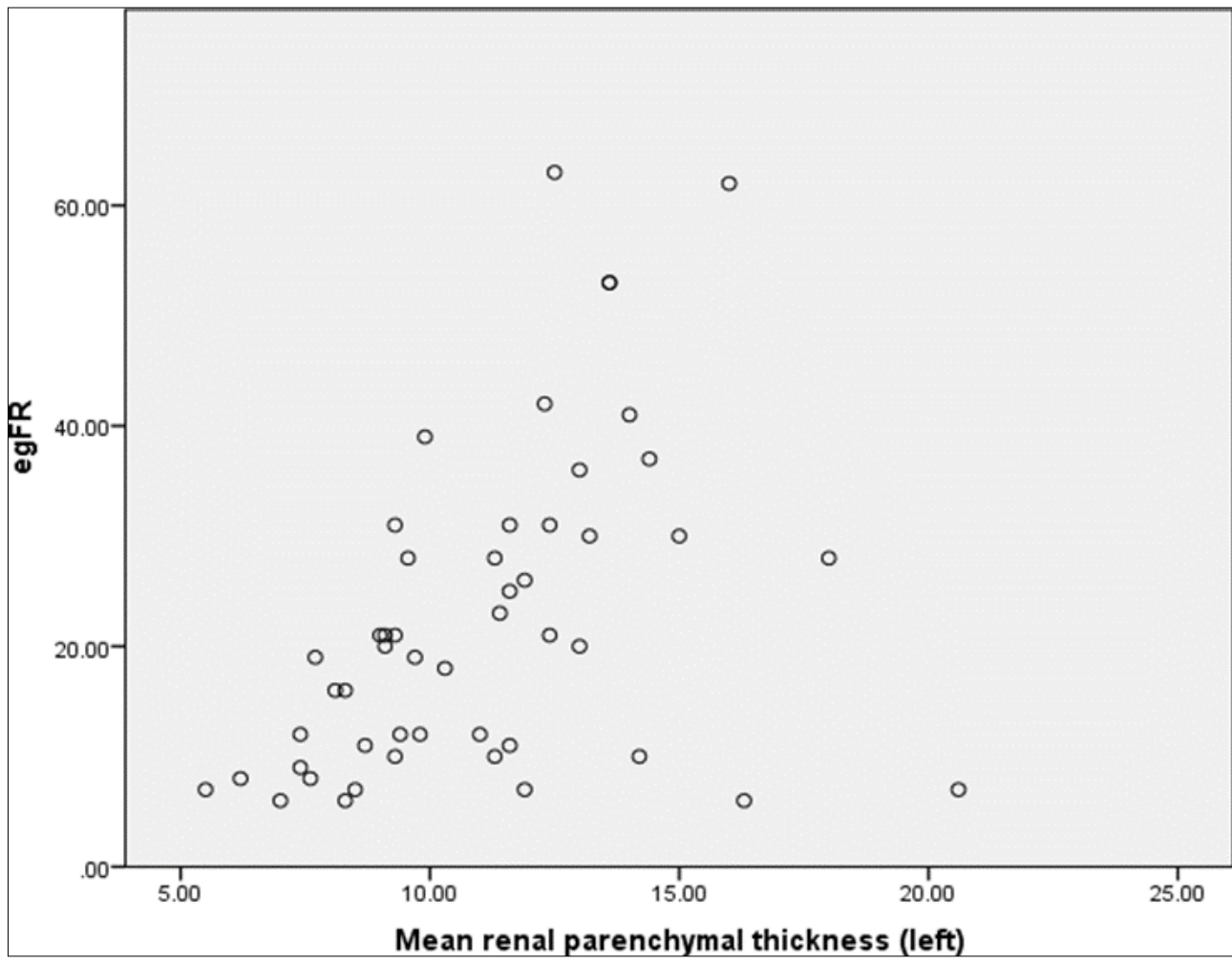

Fig 2: Correlation between eGFR and mean renal parenchymal thickness (left kidney)

\section{Results:}

In our study, 50 patients were studied, and the incidence of CKD is more commonly found in males (62\%) with predominant age group of around 41-60 yrs (64\%) followed by $61-80$ yrs $(20 \%)$. The most common cause of CKD was found to be coexisting diabetes mellitus and hypertension (44\%). 2 patients were diagnosed with chronic glomerulonephritis and 2 patients were known to have systemic lupus erythematous. The underlying cause for the renal disease was unknown in 10 cases $(20 \%)$. Majority of the patients had a normal BMI (64\%) followed by overweight $(24 \%)$, underweight $(8 \%)$ and obese $(4 \%)$.An eGFR $<15 \mathrm{ml} / \mathrm{min}$ is classified as stage $\mathrm{V},<30$ as stage IV, $<60$ as stage III, $<90$ as stage II and $>90$ as stage I. $38 \%$ patients were classified into the Stage $\mathrm{V}$ group followed by $32 \%$ in stage IV and $26 \%$ in stage III. Only $4 \%$ had near 
to normal renal function and were considered as stage II.

The mean renal parenchymal thickness was $11.2 \pm 3.01$. $68 \%$ of cases demonstrates grade II echogenicity on right side and $70 \%$ of cases with grade II echogenicity on left side i.e. the renal parenchymal echogenicity is greater than the liver/spleen with maintained cortico-medullary differentiation, followed by grade III parenchymal echogenicity with poor cortico-medullary differentiation of $14 \%$ cases on right side and $12 \%$ on left side. The mean eGFR was $22.8 \pm 15.1$ using MDRD formula. There was a statistically significant relationship between renal parenchymal thickness and eGFR using MDRD formula $(\mathrm{p}=0.002)$.

\section{Discussion}

Our study showed a statistically significant correlation between the parenchymal thickness and eGFR using MDRD formula. Emamian et al, 1993, observed renal length, width, and thickness of the kidney and its central echogenic area and the parenchymal thickness of the upper pole were measured in an age- and sex-stratified random sample of 665 volunteers. The most exact measurement of renal size is renal volume, which showed the strongest correlation with height, weight, and total body area ${ }^{[11]}$.

Levey AS et al. 1999, stated that calculating estimated glomerular filtration rate using MDRD formula is more accurate than the creatinine clearance calculated by Cockcroft-Gault formula ${ }^{[12]}$.

Beland MD et al. 2007, concluded that the mean cortical thickness measured on ultrasound is strongly correlated with the eGFR using both the CG and MDRD formula (CG, $\mathrm{p}<0.0001$, MDRD, $\mathrm{p}=0.0050)^{[13]}$.

Lucisano G et al. 2015, concluded that the ultrasonographic parameters such as pole- to-pole renal length and parenchymal thickness (taken according to standardize rules and corrected for body height) are correlated with the glomerular filtration rate that makes renal ultrasound a reliable tool for the assessment of Chronic Kidney Disease [4].

Shivashankara VU et al. 2016, stated that the renal sonographic parameters and eGFR using MDRD formula has good correlation and the renal status in the CKD patients can be more accurately reported ${ }^{[10]}$.

Yaprak M et al, 2016, stated that the kidney length and parenchymal thickness is correlated with the clinical eGFR value, whereas the parenchymal echogenicity is not always correlated with eGFR and therefore parenchymal echogenicity alone is not a good parameter for the evaluation of kidney disease. The ultrasonographic CKD score which has been calculated from the mean kidney length, parenchymal thickness and parenchymal echogenicity grading is useful in identifying stage 3-5 compared to stage $1 \& 2$ of CKD ${ }^{[14]}$.

Ahmed S et al. 2019, conducted a study in comparison of ultrasonographic findings in CKD patients with serum creatinine value, they found a strong positive correlation between the renal cortical echogenecity and the serum creatinine $(\mathrm{r}=0.915, \mathrm{P}=0.0005)$. They also stated that the mean parenchymal thickness showed positive correlation with the serum creatinine and renal length is negative correlated with the serum creatinine. Increases serum creatinine reflects increase in renal cortical echogenicity, therefore it is important to include USG parameters especially cortical echogenicity grading in evaluating the severity of CKD ${ }^{[15]}$.

\section{Conclusion}

Ultrasound is a noninvasive, low cost, easily available imaging modality in the patients with abnormal renal function tests, clinically eGFR helps to identify severity of disease and USG plays a vital role in demonstration of renal parenchymal changes. The renal length, parenchymal thicknesses are decreasing and parenchymal echogenicity increases with the progression of the renal disease. Renal parenchymal thickness appears closely related to the eGFR using MDRD formula. Therefore, renal parenchymal thickness should be considered in routine ultrasound reporting for the patients with chronic kidney disease who are not on dialysis.
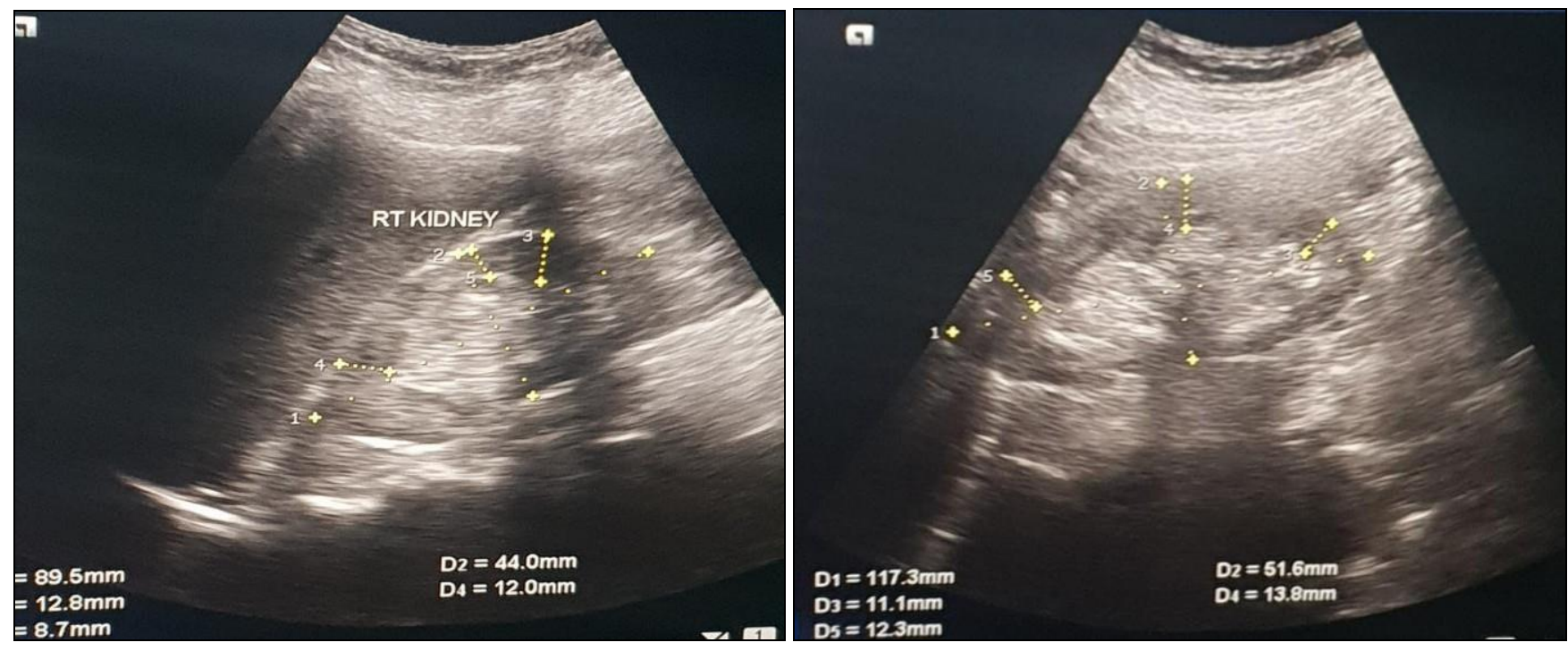

Fig 3: Case 1, Renal length and renal parenchymal thickness (at upper, mid and lower pole) of right and left kidneys 

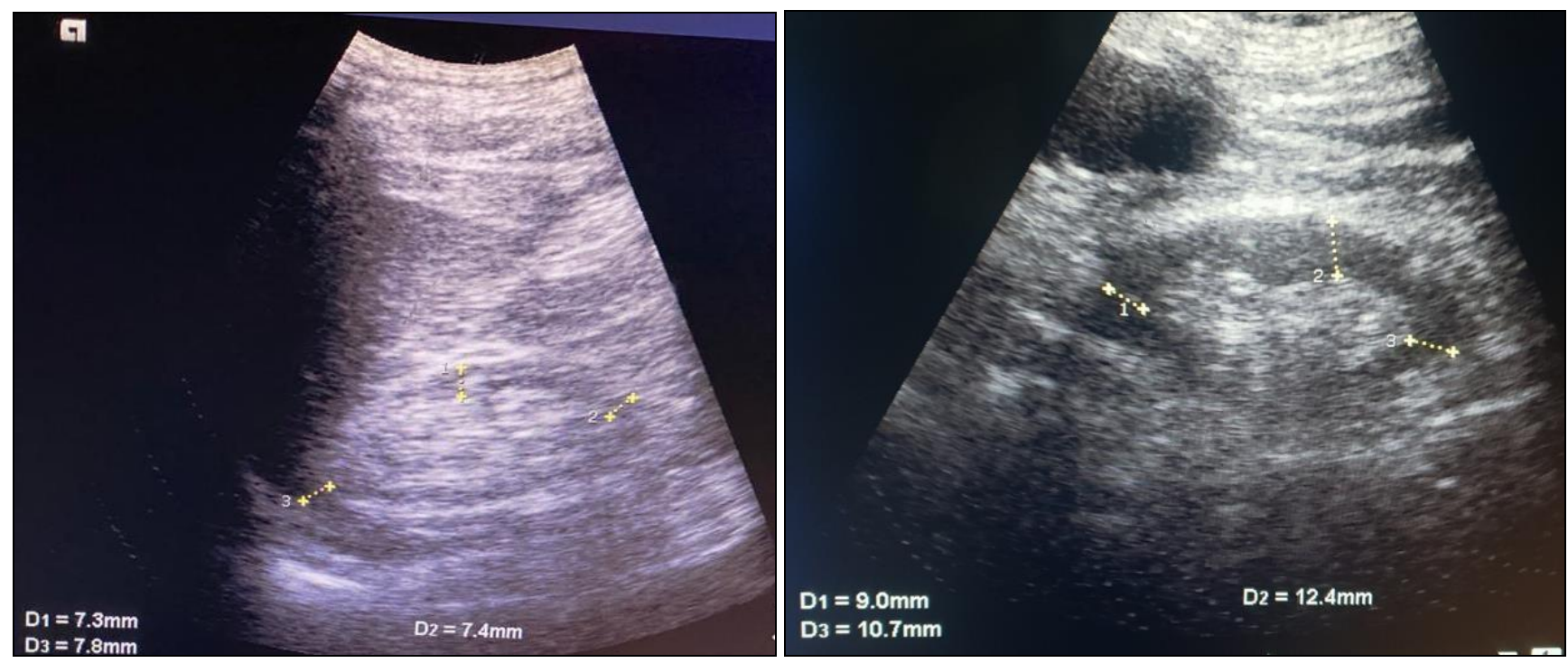

Fig 4: Case 2, Renal parenchymal thickness (at upper, mid and lower level) of right and left kidneys.

\section{References}

1. Pei XL, Xie JX. Functional MRI: evaluation of chronic kidney diseasewith perfusion imaging. Acad Radiol 2009;16:88-95.

2. Thomsen HS. Nephrogenic systemic fibrosis: a serious late adversereaction to gadodiamide. Eur Radiol 2006;16:2619-2621.

3. Gleeson TG, Bulugahapitiya S. Contrast-induced nephropathy. AJRAm J Roentgenol 2004;183:16731689

4. Lucisano G, Comi N, Pelagi E, Cianfrone P, Fuiano L, Fuiano G. Can Renal Sonography Be a Reliable Diagnostic Tool in the Assessment of Chronic Kidney Disease? Journal of Ultrasound in Medicine 2015;34(2):299-306.

5. Hricak H, Cruz C, Romanski R, Uniewski MH, Levin NW, Madrazo BL, et al. Renal parenchymal disease: sonographic-histologic correlation. Radiology 1982;144(1):141-7.

6. Cheong B, Muthupillai R, Rubin MF, Flamm SD. Normal values for renal length and volume as measured by magnetic resonance imaging. Clinical journal of the American Society of Nephrology 2007;2(1):38-45.

7. Khati NJ, Parghi CR, Brindle KA. Multimodality Imaging of the Essure Permanent Birth Control Device: Emphasis on Commonly Overlooked Abnormalities. American Journal of Roentgenology 2011;196(5):W648-58.

8. Moghazi S, Jones E, Schroepple J, Arya K, Mcclellan $\mathrm{W}$, Hennigar RA, et al. Correlation of renal histopathology with sonographic findings. Kidney International 2005;67(4):1515-20.

9. Chen QK, He F, Feng XR, et al. Correlation of Doppler parameters with renal pathology: a study of 992 patients. Exp Ther Med 2014;7:439-442.

10. Shivashankara VU, Shivalli S, Santhosh BHP, et al. A Comparative Study of Sonographic Grading of Renal Parenchymal Changes and Estimated Glomerular Filtration Rate (eGFR) using Modified Diet in Renal Disease Formula. J Clin Diagn Res 2016;10(2):TC09TC11.

11. Emamian SA, Nielsen MB, Pedersen JF, Ytte L. Kidney dimensions at sonography: correlation with age, sex, and habitus in 665 adult volunteers. American Journal of Roentgenology 1993;160(1):83-6.

12. Levey AS, Bosch JP, Lewis JB, Greene T, Rogers N, Roth D. A more accurate method to estimate glomerular filtration rate from serum creatinine: a new prediction equation. Annals of internal medicine 1999;130(6):461-70.

13. Beland MD, Walle NL, Machan JT, Cronan JJ. Renal cortical thickness measured at ultrasound: is it better than renal length as an indicator of renal function in chronic kidney disease?. American Journal of Roentgenology 2010;195(2):W146-9.

14. Yaprak M, Cakir O, Turan MN, et al. Role of ultrasonographic chronic kidney disease score in the assessment of chronic kidney disease. International Urology and Nephrology 2017;49(1):123-31.

15. Ahmed S, Bughio S, Hassan M, Lal S, Ali M. Role of Ultrasound in the Diagnosis of Chronic Kidney Disease and its Correlation with Serum Creatinine Level. Cureus 2019;11(3). 